Results 134 CT-positive patients were diagnosed before and 116 were diagnosed after service introduction. Of these, 2 $(1.5 \%)$ and $8(6.9 \%)$ respectively were treated elsewhere and excluded. Average time to treatment decreased from 6.5 to 4.1 days $(\mathrm{p}=0.09)$. When we excluded patients treated as contacts of CT (before NAAT result was available) the time to treatment decreased from 8.7 to 5.1 days $(\mathrm{p}<0.001)$. The reduction in time to treatment decreased significantly more in women; 11.3 to 6.1 days $(\mathrm{p}<0.001)$ than men; 6.8 to 4.7 days $(\mathrm{p}=0.27)$.

Conclusions Introduction of a rapid STI service significantly reduced time from testing to treatment of CT. Availability of microscopy for symptomatic men allows immediate treatment of urethritis, a common presentation of CT in men. This probably explains why men were treated earlier than women. Earlier treatment is expected to reduce both asymptomatic CT transmission, and risk of CT complications, particularly in women.

\section{P340 POTENTIAL EFFECTS OF ANTIBACTERIAL MOUTHWASH ON NEISSERIA GONORRHOEAE TRANSMISSION AMONG MEN WHO HAVE SEX WITH MEN: A MATHEMATICAL MODELLING STUDY}

${ }^{1,2,3} \mathrm{XXu}$, ${ }^{2,3,4} \mathrm{E}$ Chow, ${ }^{1} \mathrm{M}$ Shen, ${ }^{1} \mathrm{Z}$ Zou, ${ }^{5} \mathrm{C}$ Wang, ${ }^{1,2,3} \mathrm{~J}$ Ong, ${ }^{1,2,3} \mathrm{C}$ Fairley, ${ }^{1,2,3} \mathrm{~L}$ Zhang ${ }^{*}$ ${ }^{\prime} X i ' a n$ Jiaotong University, Xi'an City, China; ${ }^{2}$ Melbourne Sexual Health Centre, Melbourne, Australia; ${ }^{3}$ Monash University, Melbourne, Australia; ${ }^{4}$ The University of Melbourne, Melbourne, Australia; ${ }^{5}$ Zhengzhou University, Zhengzhou City, China

\subsection{6/sextrans-2021-sti.390}

Background Three randomised controlled trials have either reported that mouthwash may increase the susceptibility of the oropharynx to Neisseria gonorrhoeae or potentially decrease its transmissibility. We modelled these potential impacts on gonorrhoea incidence.

Methods We calibrated a susceptible-infected-susceptible compartmental model to examine the effectiveness of antibacterial mouthwash on the transmission of Neisseria gonorrhoeae in men who have sex with men (MSM). Four scenarios include: (1) mouthwash had no effect; (2) mouthwash increased the susceptibility of the oropharynx to Neisseria gonorrhoeae; (3) mouthwash reduced the transmissibility of Neisseria gonorrhoeae from the oropharynx; (4) we combined the effect of mouthwash from scenarios 2 and 3.

Results Under scenario 1, the overall incidence of gonorrhoea was 44 (95\% CI: 37-50)/100 person-years (PY). Site-specific incidence/100 PY at the oropharynx, anorectum and urethra were 26 (22-31), $9(8-11)$ and $8(5-12)$. Under scenario 2, with between $20-80 \%$ mouthwash coverage in the MSM population, the incidence increased at all three anatomical sites by between $7.4 \% \quad(5.9-60.8 \%)$ and $136.6 \%$ (108.1-177.5\%). Under scenario 3, with the same coverage, the incidence decreased at all anatomical sites by between $11.6 \%$ (10.2$13.5 \%$ ) and $99.8 \%$ (99.2-100\%). Under scenario 4, changes in the incidence depended on the efficacy of mouthwash on the transmissibility and susceptibility with both leading to large increases of nearly $130 \%$ or large declines of almost $100 \%$.

Conclusions The effect of mouthwash on gonorrhoea incidence is largely predictable depending on whether it increases the susceptibility to or reduces the transmissibility of Neisseria gonorrhoeae, highlighting an urgent need for further empirical investigation.
P341

PREDICTING THE DIAGNOSIS OF HIV AND SEXUALLY TRANSMITTED INFECTIONS AMONG MEN WHO HAVE SEX WITH MEN USING MACHINE LEARNING APPROACHES

${ }^{1,2} \mathrm{~N}$ Medland, ${ }^{1,2,3} \mathrm{C}$ Fairley, ${ }^{4} \mathrm{~J} \mathrm{Wu},{ }^{4} \mathrm{X} W \mathrm{U},{ }^{1,2,4} \mathrm{E}$ Chow, ${ }^{1,2,3} \mathrm{XXu},{ }^{2} \mathrm{Z} \mathrm{Ge},{ }^{5} \mathrm{X}$ Zhuang, ${ }^{1,2,3}$ L Zhang*. ${ }^{2}$ Melbourne Sexual Health Centre, Melbourne, Australia; ${ }^{4}$ Monash University, Melbourne, Australia; 'Xi'an Jiaotong University, Xi'an, China; ${ }^{3} T$ The University of Melbourne, Melbourne, Australia; ${ }^{5}$ Nantong University, Nantong City, China

\subsection{6/sextrans-2021-sti.391}

Background We aimed to develop machine learning models and evaluate their performance in predicting HIV and sexually transmitted infections (STIs) diagnosis based on a cohort of Australian men who have sex with men (MSM).

Methods We collected clinical records of 21,273 Australian MSM during 2011-2017. We compared accuracies for predicting HIV and STIs (syphilis, gonorrhoea, chlamydia) diagnosis using four machine learning approaches against a multivariable logistic regression (MLR) model.

Results Machine learning approaches consistently outperformed MLR. Gradient boosting machine (GBM) achieved the highest area under the receiver operator characteristic curve for HIV (76.3\%) and STIs (syphilis, 85.8\%; gonorrhoea, 75.5\%; chlamydia, 68.0\%), followed by extreme gradient boosting (71.1\%, 82.2\%, 70.3\%, 66.4\%), random forest $(72.0 \%$, $81.9 \%, 67.2 \%, 64.3 \%)$, deep learning $(75.8 \%, 81.0 \%, 67.5 \%$, $65.4 \%)$ and MLR (69.8\%, 80.1\%, 67.2\%, 63.2\%). GBM models demonstrated the ten greatest predictors collectively explained $62.7-73.6 \%$ of variations in predicting HIV/STIs. STIs symptoms, past syphilis infection, age, time living in Australia, frequency of condom use with casual male sexual partners during receptive anal sex and the number of casual male sexual partners in the past 12 months were most commonly identified predictors.

Conclusions Machine learning approaches are advantageous over multivariable logistic regression models in predicting HIV/STIs diagnosis.

\section{P342 THE IMPACT OF COVID-19 ON THE SEXUAL HEALTH OF YOUTH IN THE NETHERLANDS}

H De Graaf, F Joemmanbaks*, J Heijne, S Meijer, J Polet. Rutgers, Utrecht, The Netherlands

\subsection{6/sextrans-2021-sti.392}

Background Coronavirus disease (COVID-19) changed people's life drastically, due to restrictions to reduce transmission such as social distancing and the limited number of social contacts. The objective is to gain insight in the impact of the COVID19 pandemic on the sexual health of youth in the Netherlands.

Methods We conducted two cross sectional surveys targeting Dutch youth aged 16-20 year old during the pandemic. Recruitment occurred via social media and a youth sexual health website (sense.info). Both studies included a questionnaire about dating, relationships, sexual- and help seeking behavior and mental health. We identified 4 different time periods: (i) 6 months before the pandemic (pre pandemic), (ii) first lockdown, (iii) between lockdowns, and (iv) second lockdown.

Results The samples consisted of 5218 and 4091 participants. The study showed that less singles had sex during the first $(40 \%)$ and second (52\%) lockdown period compared to pre 
lockdown (67\%). Singles who continued to have sex more often reported casual regular partner during the first (58\%) and second lockdown (60\%), than pre lockdown (28\%) and between lockdowns (33\%). STI testing uptake decreased from $9 \%$ pre-lockdown to $1 \%$ during the first and $3 \%$ during the second lockdown. Self-tests were used almost twice as often during the pandemic (13\%) as before (7\%). Among the reasons for not testing, corona-related reasons (e.g., STI clinics are closed, or health care providers are too busy) decreased between the first (47\%) and second lockdown (27\%). Furthermore, youths mental health has weakened since the first lockdown up until the end of 2020 .

Conclusion COVID-19 pandemic significantly impacts the sexual health of Dutch youth, especially during lockdown periods, but also in periods of relaxation of pandemic control measures. Therefore, it is extremely important for healthcare providers to make their facilities accessible and find for alternatives for face-to-face testing.

\section{P345 TEST AND TREAT, OR TEST AND WAIT?: MANAGING SEXUAL PARTNERS AT RISK FOR STI}

${ }^{1} \mathrm{~F}$ van den Heuvel ${ }^{*},{ }^{1} \mathrm{E}$ Tahar-Kooijman, ${ }^{1,2} \mathrm{H}$ de Vries, ${ }^{1} \mathrm{~T}$ Heijman. ${ }^{1}$ Public Health Service of Amsterdam, Department of Infectious Diseases, Amsterdam, The Netherlands; ${ }^{2}$ Department of Dermatology, Amsterdam Institute for Infection and Immunity (AlandII), Amsterdam University Medical Centers, location Academic Medical Centre, University of Amsterdam, Amsterdam, The Netherlands

\subsection{6/sextrans-2021-sti.393}

Background Treatment of sexual partners notified for Chlamydia trachomatis(CT) or Neisseria gonorrhoeae (NG) is needed to prevent ongoing transmission. To prevent the emergence of Anti-Microbial resistance, antibiotics should be used prudently. Therefore, in 2020, the STI clinic of the Public Health service of Amsterdam (STI clinic) went from treating all confirmed notified sexual partners (before test results) to a selection of the notified partners i.e. only those who received confirmed notification from a steady sex partner, with whom they had sex in the last 2 months. We evaluated STI outcomes before and after implementation of this policy.

Methods Routine data from confirmed notified partners attending the clinic for CT/NG from 01-09-2019 to 01-032020 were compared with similar data from 01-03-2020 to 01-10-2020.

Results CT: of the 132 partners notified before policy change, $64 \%(84 / 132)$ had a negative test result, all received treatment before results were available. After policy change 71\% (163/ 230) had a negative result, $17 \%(39 / 230)$ of them received treatment before results were available and 14\% (32/230) positive for CT had to return to the clinic to receive treatment.

NG: of the 131 partners notified before policy change $72 \%(94 / 131)$ had a negative test result, all received treatment before results were available. After policy change 71\% (215/ $304)$ had a negative test result, 27\% (59/215) of them received treatment before results were available and 57\% (51/ 89 ) of the patients positive for NG had to return to the clinic for treatment.

Discussion Unnecessary treatment with antibiotics decreased substantially when introducing the new policy. A limited amount of partners had a delay in treatment and had to return to the clinic. The cost of a second visit and the delay to treatment will be assessed in order to improve services and decrease the risk of distribution of CT/NG by untreated partners.
P346 REACHING SEXUAL PARTNERS AT RISK FOR STI THROUGH SOCIAL MEDIA

${ }^{1}$ E Tahar-Kooijman*, 'F van den Heuvel, ${ }^{1,2} \mathrm{H}$ de Vries, ${ }^{1} \mathrm{~T}$ Heijman. ${ }^{1}$ Public Health Service of Amsterdam, Department of Infectious Diseases, Amsterdam, The Netherlands; ${ }^{2}$ Department of Dermatology, Amsterdam Institute for Infection and Immunity (AlandII), Amsterdam University Medical Centers, location Academic Medical Centre, University of Amsterdam, Amsterdam, The Netherlands

\subsection{6/sextrans-2021-sti.394}

Background At the STI clinic of the Public Health service of Amsterdam (STI clinic) partner notification (PN) seems successful in the early diagnosis and treatment of STI's in sexual partners. Together with the healthcare staff, and upon consent of the index patient, sexual partners at risk are identified and the PN methods discussed: patient or provider referral, by phone, mail or an online PN service. However, in a substantial number of cases, contact details are missing because of the anonymity of the partner, e.g in case of social media (SM) use. We therefor explored the willingness and opinion of patients to use SM for online PN.

Methods From December 172020 to February 12021 we invited all patients visiting the STI clinic for treatment to fill in an anonymous online questionnaire on the use of SM for online PN: 1) using SM for meeting and notifying sexual partners, and 2) to be approached themselves as a notified partner. Lastly, they were asked to comment on a series of potential texts messages to be used for online PN.

Results 180 patients participated in the study: 68\% males, $32 \%$ females and $0,6 \%$ transgender persons. $80 \%$ indicated they met a sexual partner via SM and $57 \%$ of them only had SM contact details. 59\% would like to use a message via SM for PN purposes, and $73 \%$ would want to receive a PN via SM. $40 \%$ would want to be informed when involving HIV. The majority would choose a format containing the logo of the public health service and the online PN service because this appeared more trustworthy.

Discussion Preliminary analyses show the potential of online PN through SM. However safety and trustworthyness are concerns when developing the intervention and effectiveness should be analysed.

\section{P347 DEVELOPMENT AND EXTERNAL VALIDATION OF A PROGNOSTIC MODEL FOR SURVIVAL OF PEOPLE LIVING WITH HIVIAIDS INITIATING ANTIRETROVIRAL THERAPY}

1,2J Wang, ${ }^{3} \mathrm{~T}$ Yuan*, ${ }^{4} \mathrm{H}$ Ding, ${ }^{4} \mathrm{~J} \mathrm{Xu},{ }^{1} \mathrm{~W}$ Keusters, ${ }^{5} \mathrm{X}$ Ling, ${ }^{3} \mathrm{~L} F u,{ }^{3} \mathrm{Q} Z \mathrm{Zhu},{ }^{5} \mathrm{Q} \mathrm{Li},{ }^{5} \mathrm{X}$ Tang, ${ }^{5} \mathrm{~W}$ Cai, $6,7,8,9 \mathrm{H}$ Hong, ${ }^{5} \mathrm{~L} \mathrm{Li}, 3,10 \mathrm{H}$ Zou. ${ }^{1} J u l i u s$ Center for Health Sciences and Primary Care, University Medical Center Utrecht, Utrecht, The Netherlands; ${ }^{2}$ Division of Pharmacoepidemiology and Clinical Pharmacology, Utrecht Institute for Pharmaceutical Sciences, Utrecht University, Utrecht, The Netherlands; ${ }^{3}$ School of Public Health (Shenzhen), Sun Yat-sen University, Shenzhen, China; ${ }^{4}$ NHC Key Laboratory of AIDS Immunology, Department of Laboratory Medicine, First Affiliated Hospital of China Medical University, Shenyang, China; ${ }^{5}$ Eighth People's Hospital, Guangzhou Medical University, Guangzhou, China; ${ }^{6}$ Key Laboratory of AIDS Immunology of National Health Commission, Department of Laboratory Medicine, the First Affiliated Hospital of China Medical University, Shenyang, China; ${ }^{7}$ National Clinical Research Center for Laboratory Medicine, the First Affiliated Hospital of China Medical University, Shenyang, China; ${ }^{8}$ Collaborative Innovation Center for Diagnosis and Treatment of Infectious Diseases, Hangzhou, China; ${ }^{9} \mathrm{Key}$ Laboratory of AIDS Immunology, Chinese Academy of Medical Sciences, Shenyang, China; ${ }^{10}$ Kirby Institute, the University of New South Wales, Sydney, Australia

10.1136/sextrans-2021-sti.395 